\title{
An Acceptability Study Of A Personal Portable Device Storing Critical Health Information To Ensure Treatment Continuity Of Home-Dwelling Older Adults In Case Of A Disaster
}

This article was published in the following Dove Press journal:

Patient Preference and Adherence

\begin{abstract}
Andreas Hein Willius (D)
Marisa Torres Hidalgo ${ }^{2}$

Pablo Arroyo Zuñiga'

Margarita Quezada Venegas (ID)

Christian Arriagada Díaz ${ }^{3}$

Eduardo Valenzuela Abarca ${ }^{4}$

Ernesto San Martín Gutierrez ${ }^{5}$

Paula Bedregal (iD) 6

'Project DEPPAS-FONDEF

(IDI7AM0038), Faculty of Medicine,

Pontifical Catholic University of Chile,

Santiago, Región Metropolitana, Chile;

${ }^{2}$ Faculty of Medicine, Pontifical Catholic

University of Chile, Santiago, Región

Metropolitana, Chile; ${ }^{3}$ School of Social

Work, Faculty of Psychology, Universidad

San Sebastián, Santiago, Chile;

${ }^{4}$ Department of Internal Medicine,

Geriatric Program, Faculty of Medicine,

Pontifical Catholic University of Chile,

Santiago, Región Metropolitana, Chile;

${ }^{5}$ Interdisciplinary Laboratory for Social

Statistics, Faculty of Mathematics,

Pontifical Catholic University of Chile,

Santiago, Región Metropolitana, Chile;

${ }^{6}$ Department of Public Health, Faculty of

Medicine, Pontifical Catholic University

of Chile, Santiago, Región Metropolitana,

Chile
\end{abstract}

Correspondence: Andreas Hein Willius Departamento de Salud Pública, Escuela de Medicina, Pontificia Universidad

Católica de Chile, Diagonal Paraguay 362,

Santiago, Región Metropolitana, Segundo

Piso, Chile

Tel +56956240699

Email aahein@uc.cl
Aims: DEPPAS ("Dispositivo Electrónico Personal y Portable en Salud" or Personal, Portable Electronic Health Device in English) is a portable device in form of a bracelet that allows storing electronic health records of older adults experiencing chronic illnesses. The device seeks to support the vital sustainability of older adults by storing critical health information when electronic or paper records have been lost as a consequence of a disaster. Older adults are particularly vulnerable to experience negative consequences in this context. The present study explores the end-user acceptability of DEPPAS in order to inform the next design stages of the device.

Methods: Twenty home-dwelling urban male and female older adults enrolled in a chronic health management program were invited to participate in two focus groups. A prototype of DEPPAS was presented and reactions to health service disruption scenarios were explored. Focus groups were transcribed. Content analysis based on the Technology Acceptance Model was conducted.

Results: Older adults are acutely aware of their vulnerable health status. Participants report overall positive reactions to DEPPAS. The device was associated with feelings of relief and an increased sense of control over their health management. DEPPAS is perceived as useful, usable, and safe. Even though concerns regarding confidentiality were raised, benefits are perceived as more relevant than potential risks. Participants agree that its usefulness could be extended beyond disaster situations to everyday health care management. Implications for future development and limitations are discussed.

Conclusion: The conceptual design DEPPAS shows a high level of acceptability by this end user and a high potential to be integrated with other complementary technologies (e.g. GPS, medication reminders) that could significantly contribute to improving health management in disaster situations.

Keywords: wearable health devices, portable electronic health records, acceptability, older adults

\section{Background}

Disasters can affect the life, health, assets and the environment of the population, beyond the capability of institutions to respond efficiently. ${ }^{1}$ Globally, climate-related natural disasters have been increasing. The nature of harm resulting from a natural disaster is shaped by a combination of natural hazards, physical, economic, socialenvironmental vulnerability and the capability to respond to them. ${ }^{2}$ Due to geographical conditions, most Chilean settlements/facilities are exposed to natural hazards (i.e. earthquakes, flooding). ${ }^{3}$ 
Older adults are among the most affected by disasters showing the highest risk of experiencing adverse effects. Older adults show increased rates of physical frailty (e.g. chronic diseases, impaired functioning), social frailty (socioeconomic conditions), limited capability to respond to external shocks and exposure to abuse. ${ }^{4-6}$

New responses to disaster consequences are needed to reduce negative impacts on older adults. In this context, information management constitutes a key element to tackle identified health needs during an emergency. ${ }^{7}$

Harm level of a natural disaster is directly related to the level of vulnerability of people, families and communities at the moment of the event. ${ }^{8}$ For example, during the Hurricane Katrina's aftermath, nearly half of the visits to emergency treatment sites were related to chronic diseases. Treatment interruption of these conditions may have serious health consequences. ${ }^{9}$ Improving preparedness to manage chronic diseases in a post-disaster context is thus a key issue. ${ }^{10}$

In Chile, it is not uncommon for natural disasters to lead to power outages or the destruction of infrastructure like health centres leading to health information to be unavailable. This hampers first responder teams' capability to make informed clinical decisions to provide appropriate health services. Older adults may experience difficulties in remembering details about their health status, (i.e. treatment, medication dosage) and are unable to help. Therefore, timely access to health records is a key aspect of this problem.

\section{Personal Portable Health Records, Wearable Health Devices, And Disaster Preparedness For Older Adults}

New developments in health information technology (e.g. wearable health devices and portable health records) have been focusing on improving the effectiveness and efficiency of health services. ${ }^{11,12}$

Most wearable health devices (WHD) focus on recording and monitoring information (signs and symptoms) of patients associated with their health status (e.g., hours of sleep, exercise, glucose, blood pressure, blood level oxygen, even geo-localization). Wearables also can remind medication administration schedules, among other applications. ${ }^{13}$ Through such devices, patients are expected to improve their self-management strategies. ${ }^{14}$ WHD have been used to support lifestyle changes ${ }^{14}$ tracking (e.g. persons with Alzheimer's disease), ${ }^{15}$ pre-post-natal health care ${ }^{5}$ or managing chronic diseases. ${ }^{16}$
Portable health records (PHR) seek to empower patients, ${ }^{17}$ to increase health understanding, ${ }^{18}$ and to enhance communication between health care professionals and patients. ${ }^{17}$ Applications may range from electronic medical records ${ }^{19}$ to web-based information sources. ${ }^{20}$ Availability of information may help to improve preparedness to manage emergencies.

Newer developments in health information technology and WHD have been leaning on the widespread penetration of mobile devices to develop interventions based on reminders, access to information and follow-up techniques to address a wide range of issues. ${ }^{21,22}$

\section{Acceptability And The Design Of Medical Devices}

Designing mobile health devices may be a challenging process involving several phases. ${ }^{11}$ It is recommended to always conduct extensive research on the "use" and the "user" of the designed device. ${ }^{23}$ Medical health devices that will be used in unsupervised settings should adhere to high standards of comfort and attention to human factors in order to facilitate technology acceptance, reduce gadget intolerance and enhance the user experience. ${ }^{24}$

The most extensively researched model to explain user acceptability is known as the "technology acceptance model (TAM)". ${ }^{25,26}$ TAM states that perceived usefulness and perceived ease of use of technology are key predictors of acceptability (attitude towards technology) and actual use of technology. With wide empirical support, ${ }^{27}$ TAM has also been extended by including additional predictors such as gender, culture, and experience, among others. ${ }^{28}$ The present study proposes an extension to TAM to address the acceptability of WHD.

In this context, acceptability of WHD is thought to be affected by the following:

(i) Perceived usefulness: believing that a particular WHD can help to address a problem of interest) ${ }^{14,25,26,29,30}$

(ii) Perceived ease of use: believing that WHD is easy to use and understand ${ }^{17,25,26,31}$

(iii) Usability: believing that WHD is enjoyable to use $\mathrm{e}^{15,20}$ in terms of aesthetics, ${ }^{24}$ comfort ${ }^{24,32}$ social norms (how others will react to it) and image (believing that the WHD will enhance social status) $)^{24,32}$

(iv) Perceived safety/privacy of information ${ }^{17,18,32}$ and

(v) Perceived cost. ${ }^{29}$ 
From this perspective, the actual use of a device is expected to depend on its acceptability by the user. ${ }^{14}$

\section{The Present Study: Acceptability Of} DEPPAS ("Dispositivo Electrónico Personal y Portable en Salud" translates as Personal, Portable Electronic Health Device)

DEPPAS was developed to support the vital sustainability of older adults when access to centralized health records has been lost (i.e. infrastructure destruction, power outage and communication loss). DEPPAS aims to ensure that health workers (i.e. disaster first responders) can access critical health information to make informed clinical decisions for older adults. In addition, this information needs to be protected to ensure confidentiality.

DEPPAS is composed of three elements:

- A personal portable device (PPD): In form of a wrist band (see figure), the PPD has a hypoallergenic rubber exterior that contains an autonomous memory capable of storing encrypted health information (e.g. acute and chronic diseases, life-threatening conditions, disabilities, medications taken, among others). It requires no power, and information can only be read and updated using a specific readerwriter device (RWD).

- A reader-writer device (RWD): The RWD can retrieve and update the information that has been stored in the personal portable device. Information can be displayed on its LCD screen. The device operates using radio frequency (RFID) as a means of transmitting and receiving data. It requires no internet access and operates on a rechargeable battery. Both are critical features if DEPPAS is expected to be useful in a post-disaster context. The reader writer device would be operated by authorized health workers (e.g. first responders).

- A centralized data storage system: RWD can interact with centralized storage systems like health care center databases, where said system comprises a central server. This allows to keep the information updated.

DEPPAS seeks to contribute to the disaster preparedness of older adults (see Figure 1).

Figure 1: DEPPAS' components include a personal portable device (PPD) in form of a wristband with an autonomous memory to store encrypted health

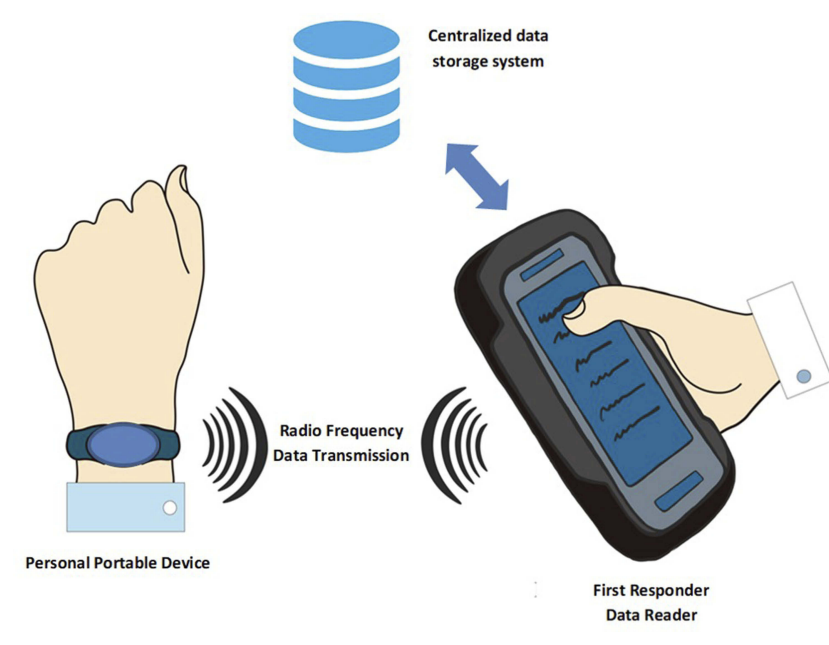

Figure I DEPPAS' components.

information; a reader-writer device (RWD) that can retrieve and update PPD information; and a centralized data storage system (i.e. centralized server). The RWD interacts with the centralized data storage system to keep the information updated.

The present paper focuses on exploring the understanding of the concept of DEPPAS in general and the acceptability of the PPD that will be used by older adults. Factors that could facilitate or hinder its use by the target population were identified. Study results were expected to guide the development of the definitive PPD prototype. Acceptability of the RWD by health professionals will be explored in a separate study.

\section{Materials And Methods Data Collection}

An exploratory qualitative study based on focus groups was implemented. ${ }^{33}$ Two sessions lasting 90 minutes where held. One including only male older adults and one including only female older adults. Each group was facilitated by two researchers, one guiding the conversation and the other taking notes and asking follow-up questions. Discussion was guided by an open-ended questionnaire. Each session was divided into three moments:

*Introduction: Researchers and participants introduce themselves to the group. Research aims are explained.

*Presentation of disaster scenarios: Three hypothetical situations depicting how different types of natural events may cause their health records to become unavailable (for example, outage, fire) are presented. These were designed to help participants to understand the problems that 
DEPPAS intends to solve. The last hypothetical situation introduces an example of how DEPPAS may help to resolve these situations. Each time, reactions from the group are explored.

*DEPPAS Evaluation: DEPPAS is explained in more detail. Participants are asked to assess its perceived attributes. DEPPAS' PPD prototypes were distributed for the participants to try them out.

\section{Sample}

Twenty self-sufficient urban home-dwelling older adults (65 + years) participated in the Chronic Care Program of one public health center located in the south-eastern part of Santiago de Chile. At this stage of the development process was considered necessary to exclude older adults that may have difficulties to participate in group dynamics (for example adults showing signs of cognitive impairment). It was decided to firstly examine acceptability in general older adult population; thus, participants did not necessarily have experiences with disaster situations.

The study was approved by the Scientific and Ethical Committee of the Pontifical Catholic Pontifical University of Chile. As the study presents a minimal risk for the participants, the requirement to obtain written informed consent was waived. Participants were verbally asked to accept to participate in the study.

\section{Analysis}

Focus groups were transcribed. Content analysis based on the proposed variation of the "Technology Acceptance Model" was conducted. Analysis was conducted by three authors (AH, MT, MQ) and discussed in research meetings with the full team.

\section{Results}

Given their health status, participants report the extensive experience as a user of the local health system. Participants seem acutely aware of their frailty expressing feelings of vulnerability. Managing their health is a clear source of concern that requires investing in a considerable effort to comply with prescribed treatments.

In my case, I have to take 4 different medicines ... to memorize them and their dosage it's complicated ... (Man)

\section{Initial Reactions}

When confronted with fictional disaster scenarios disrupting health services due to health record unavailability, most of the participants react with anger and disbelief followed by rising anxiety when imagining how to deal with the situation.

Technology dies and everything dies (Man)

And how will I be able to remember all the medicines I'm taking? (Woman)

Although the scenarios were fictitious, several participants reported having experienced similar circumstances in normal settings.

Something very similar happened here ... I told the doctor I could come with a pen drive and he could read it here, but the computer did not work either (Woman)

... the power was out, then he told me I cannot see your information, but tell me more or less what you have ... I had to tell him more or less what it was all about ... (Woman)

Remembering details about medication is perceived as the hardest task of all. Most of the participants take detailed notes on paper (or cell phone) to keep track of their medication. Others rely on a relative to help them.

When I go to the appointment with the physician, I bring a paper $(. .$.$) with everything that happens to me ... (Woman)$

Well, I usually count on my wife (laughs) ... if I fail to write something down, she remembers it ....I'm sticking to her. (Man)

\section{Perceived Usefulness}

Once DEPPAS is introduced, participants express spontaneous positive initial reactions. Most participants express feelings of relief.

A relief ... a tremendous help (Man)

I think we would feel (...) safer walking with the wristband that will record our diseases (Woman)

I think it's super positive (...) for example: if I get sick I have to resort to my neighbor and explain her the whole story (...) this way, she just can take me to the doctor ... (Woman)

As such, participants perceive it could be used beyond disasters to inform emergency health workers in case of an accident (e.g. fainting on the street, car crash or falling at home).

I, at least, would not take it off (....) (Man) 
I do not know what can happen to me, if something happens to me I will need it, for example, in the case of an accident (Woman)

Participants agree that the device should be rolled out extensively throughout the country. This would increase DEPPAS' perceived usefulness as a means to inform emergency services about their health conditions at any time and place. Broad coverage of the DEPPAS would contribute a sense of relief and safety associated with the use of DEPPAS.

It's not an obligation, but I'm thinking about the future, it should be massive (Woman)

Well, I think it would be very good (...) is it going to be a project at the country level? (Man)

If you have an accident (...)they will know what you have and the medicine they can give you. (Man)

It was also explored if the inclusion of additional functions would increase perceived usefulness. Some suggested that additional functions included a clock, alarm (to remember taking medication) and GPS to be located by relatives (mostly men). Women show a clearer preference towards simpler device only recording health information.

When confronted with a trade-off (more functions vs health records), participants prefer wide geographical coverage over added functions.

In that case I prefer to keep the simplest bracelet, and have it throughout the country (Man)

The function of health information storage is perceived as highly valuable by itself. As such, it seems to be associated with a high intent to use it. Future integration of new functions is perceived as an interesting addition by the participants. However, this does not seem critical to foster acceptability. Having said this, including an alarm (remembering medication name time and dosage) would be highly regarded by the participants.

\section{Ease Of Use}

Participants spontaneously raise many questions. Women's questions reflect concerns related to the durability of the device and preparedness of medical teams to use it. Men's questions focus more on how the device works, its cost and coverage. Participants perceive it as easy to use and prefer to keep it as it is. Adding more functions would make it more difficult to understand and to use.

\section{Usability}

Since the device is perceived as "quite usable", it seems to bring about peace of mind.

Just like the watch or a cell phone, you put on the bracelet and you can to go out, to go anywhere (Woman)

Participants stated to be generally pleased with the wrist bands aesthetic design ("looks like a "fitbit"). Participants preferred to be able to choose the color of their device, men preferring darker colors (grey, black) and rejecting pink. Women preferred varied colors and rejected black devices. Both groups gave positive assessments of bright colors such as yellow or blue. Faced with a trade-off (aesthetics vs functionality), participants clearly preferred usefulness (providing information) over aesthetics. Participants have a strong pragmatic orientation in this regard.

Regarding comfort, some doubts were expressed about using DEPPAS during night time as it might be uncomfortable. While most participants prefer a bracelet without a buckle to prevent it falling off, others would appreciate a buckle to take it off at night.

I cannot sleep with this at night, I cannot sleep with a watch, I feel like I'm oppressed. (Man)

I would not take it off (...) it has to be permanently used; I would be aware that I have to use it (Woman)

Participants expect DEPPAS to be durable, impermeable and hypoallergenic. Most perceive it is. Participants prefer smaller and thinner models.

I like adventure, I need something that cannot fall off, that one bathes with that, uses it permanently, an emergency can happen any time (Man)

Even though there was some discussion about an alternative type of devices (e.g. pendant vs wristband), most participants preferred a wristband.

Participants believe that the device would be welcomed by their family members (social norms). Particularly women feel that wearing the bracelet would allow them to open up the conversation about their health with other family members. Participants agree that they would like their relatives to know about the devices and how it can be used.

... when you use them, the questions will come, why do you walk with that wristband? What does it mean? And 
one is going to have to tell her, starting with her husband, children, then they will be calmer (Woman)

Participants perceive DEPPAS as enhancing their image as a fitbit activity tracker would. No concerns regarding the negative effect on the personal image were voiced. As discussed before, perceived usefulness still seems to be the most relevant and valued characteristic of DEPPAS.

\section{Safety/Confidentiality}

Participants show little apprehensions regarding the confidentiality of storing sensitive health information on the wristband. Concerns were voiced by women regarding the inclusion of information on sexually transmitted diseases. In the male group, concerns were voiced related to the potential misuse that companies could do if they had access to such information.

Both groups agree that even if sensitive information is stored, the potential benefits outweigh the perceived risks.

If it's in our benefit, no problem (Woman)

I believe that at this stage of our lives and with all the experience that each one has as older adults, there are no such restrictions as when one was young, but there are benefits, and in that case the benefits are positive. (Woman)

\section{Cost}

Even though it is not expected that the wristband would have a cost for the end user, participants voiced the willingness to pay up to 7,5 USD for such a wristband.

No other relevant codes related to acceptability emerged. As such, the proposed framework was deemed adequate to examine the research question. Table 1 displays a summary of the themes that have been derived from the transcripts. It also shows the frequency with which each theme is mentioned in each of the groups (male, female).

\section{Discussion}

Older adults in general comprise a heterogeneous group (varied diseases, cognitive or motor disabilities); thus, designing WHD for them may be particularly challenging. ${ }^{11,34}$ Paying attention to human factors positively influences user acceptability and use. ${ }^{24}$

The aim of the present study was to explore the end user's understanding of DEPPAS and acceptability of the personal portable device (PPD). Particular attention was paid to identify factors that could facilitate or hinder the use of the device by the target end user population.

Results suggest that participants understood the concept of DEPPAS which seemed to be associated with feelings of relief and increased sense of safety. Participants expressed high expectations regarding DEPPAS and easily imagined applications that went beyond disaster preparedness but also regular day-to-day lives. Off all factors related to acceptability (perceived usefulness, perceived ease of use, usability, safety/privacy of information; cost), the most valued attribute of the device seems to have been its perceived usefulness. Participants expressed willingness to trade other potential valued attributes (e.g. added functions alike GPS, their preferred colour) or perceived barriers (e.g. privacy of information) if the device was able to fulfil its key function to facilitate timely access to health information for medical staff.

These findings are consistent with previous literature suggesting that older adults' concerns are related primarily to ease of use and perceived usefulness. ${ }^{14,25,26,32,35-38}$ Since DEPPAS' use does not require any effort, the discussion will focus on the role of usefulness. Perceived usefulness plays a key role in technology acceptability by older adults. Older adults tend to have a positive attitude towards technology if they perceive that it can help them to improve their quality of life. ${ }^{35}$ Home-dwelling older adults particularly value health technology that allows them to enable them to remain in their homes and to keep an independent life. ${ }^{36,39}$ They also value technology that can facilitate emergency assistance in case of an accident. $^{37,38}$ Conversely, older adults may resist technology if it is perceived it will make them lose independence and autonomy. ${ }^{40}$

Although other factors seemed to have a secondary relevance, their importance should not be downplayed, especially in the case of usability (comfort). ${ }^{32}$ Participants' responses suggest that comfort may play a significant role in future use (i.e. day vs night use). If DEPPAS is going to be a device that can effectively prevent service disruption during a disaster, a key challenge will be related to participants using it consistently over time. Comfort might play a key role in this regard.

Another issue frequently raised in the WHD and PHR literature is related to confidentiality protection. ${ }^{17,18}$ Results suggest that even though concerns were raised, participants seemed to have been less worried about privacy than about usefulness of DEPPAS. This is also 
Table I Extracted Codes And Frequency There Are Mentioned In Each Of The Groups

\begin{tabular}{|c|c|c|c|c|c|}
\hline & \multicolumn{2}{|c|}{ Women } & \multicolumn{2}{|c|}{ Men } & \multirow{2}{*}{$\begin{array}{l}\text { Totals } \\
\mathbf{N}\end{array}$} \\
\hline & $\mathbf{N}$ & $\%$ & $\mathbf{N}$ & $\%$ & \\
\hline \multicolumn{6}{|l|}{ Initial reactions } \\
\hline Expresses anxiety with a fictional scenario & 7 & 46.7 & 8 & 53.3 & 15 \\
\hline Mentions difficulties in remembering medication & 5 & 23.8 & 16 & 76.2 & 21 \\
\hline Remembers similar event in real life & 7 & 87.5 & I & 12.5 & 8 \\
\hline \multicolumn{6}{|l|}{ Perceived usefulness } \\
\hline Expresses relief after DEPPAS' concept is introduced & 8 & 61.5 & 5 & 38.5 & 13 \\
\hline Suggest DEPPAS may have uses beyond emergencies & 4 & 100 & 0 & 0 & 4 \\
\hline Suggest additional functions & 4 & 50 & 4 & 50 & 8 \\
\hline Suggest extensive roll-out & 7 & 87.5 & 1 & 12.5 & 8 \\
\hline Prefer simpler device vs a multifunctional device & 6 & 66.7 & 3 & 33.3 & 9 \\
\hline Value health record information storage & 2 & 25 & 6 & 75 & 8 \\
\hline \multicolumn{6}{|l|}{ Ease of use } \\
\hline Ask about materials and durability & 8 & 100 & 0 & 0 & 8 \\
\hline Ask about how device works & 0 & 0 & 12 & 100 & 12 \\
\hline Ask about preparedness of first responders & 2 & 50 & 2 & 50 & 4 \\
\hline \multicolumn{6}{|l|}{ Usability } \\
\hline Prefer choosing colors vs colors meaning specific things & 4 & 100 & 0 & 0 & 4 \\
\hline Prefer darker colors & 0 & 0 & 5 & 100 & 5 \\
\hline Prefer brighter colors & 8 & 100 & 0 & 0 & 8 \\
\hline Prefer usefulness over aesthetics & 3 & 100 & 0 & 0 & 3 \\
\hline Prefers wristband format & 5 & 50 & 5 & 50 & 10 \\
\hline Prefers pendant format & I & 100 & 0 & 0 & 1 \\
\hline Would like relatives/friends to know how device works & 8 & 80 & 2 & 20 & 10 \\
\hline Perceive it as enhancing their image & 0 & 0 & 2 & 100 & 2 \\
\hline \multicolumn{6}{|l|}{ Safety/confidentiality } \\
\hline Voice concerns regarding confidentiality & 4 & 40 & 6 & 60 & 10 \\
\hline Favors usefulness over confidentiality & 6 & 33.3 & 12 & 66.7 & 18 \\
\hline \multicolumn{6}{|l|}{ Cost } \\
\hline Asked about price/willingness to pay & 0 & 0 & 4 & 100 & 4 \\
\hline Total & 99 & 51.3 & 94 & 48.7 & 193 \\
\hline
\end{tabular}

consistent with previous research ${ }^{36,37}$ suggesting that older adults tend to be less concerned with confidentiality than younger adults. ${ }^{32}$ Older adults may be more concerned with the technology being intrusive (i.e. getting in the way) than with privacy issues. ${ }^{36}$ Although older adults may resist some forms of monitoring technology (i.e. home-based cameras), ${ }^{37}$ they usually are willing to accept some forms of monitoring data sharing as long as this would allow identifying health problems or assistance during an emergency. Despite this, data privacy should still be a primary concern for WHD and PHR development, and measures should be taken to avoid improper use of information. ${ }^{12,21}$

Results suggest that DEPPAS may have wider applications than anticipated. In this context, it seems important to note that the observed high expectations regarding DEPPAS functionality will pose the challenge to properly inform what can and cannot be expected from the device and to reinforce its perceived usefulness to promote actual use. 


\section{Limitations And Future Directions}

Some limitations need to be acknowledged. Firstly, although DEPPAS was designed as a tool to address the health management in the aftermath of a disaster, it was considered important to first test the concept in a sample of general older adults rather than focusing only on those who have experienced a disaster situation. Thus, conclusions on its applicability and usability in a disaster situation are still preliminary. Secondly, most of the participants come from a middle class to a low class, most are educated (post-secondary education, some are professionals), and actively participate in older adult clubs and activities. These characteristics may limit the generalizability of present findings. Finally, participants did not yet have the experience of actually using DEPPAS; thus, its acceptability under actual use conditions needs to be tested. The next research stages will focus on studying acceptability after using DEPPAS in diverse older adult samples. Also, acceptability by health professionals will be explored.

\section{Conclusions}

The conceptual design DEPPAS seems to be acceptable for its end user group. In this regard, DEPPAS also appears to show the potential to be integrated with other complementary technologies (e.g. watch, medication reminders) that could significantly contribute to improve health self-management.

In sum, participants expressed disposition to use DEPPAS. Also, no relevant initial barriers could be detected in relation to the use of DEPPAS in the format in which it is currently being considered.

\section{Acknowledgments}

This research has been funded through project CONICYTFONDEF/CUARTO CONCURSO IDeA EN DOS ETAPAS, FONDEF/CONICYT 2017- ID17AM0038. The team would also like to thank Dr. Claudia Latorre Leyton (Director of CESFAM Padre Manuel Villaseca), Diego Silva Nuñez (Head of the Self-Sufficient Older Adult Program (PAMA)) and Luz María Montalva Herrera (Director of Health Services at the Corporación Municipal de Puente Alto) for their invaluable help in recruiting the study participants and facilitating access to a venue to conduct study activities.

\section{Disclosure}

Margarita Quezada Venegas reports grants and personal fees from Comisión Nacional de Investigación Científica y Tecnológica, during the conduct of the study and grants and personal fees from Comisión Nacional Científica y Tecnológica, outside the submitted work. Andreas Hein Willius, Marisa Torres Hidalgo, Pablo Arroyo Zuñiga, Christián Arriagada Díaz and Paula Bedregal report grants from Conicyt, during the conduct of the study. Marisa Torres Hidalgo and Margarita Quezada Venegas declare to have applied for a patent for DEPPAS (PCT/CL2018/ 000036) as co-inventors. This patent has been donated to Pontificia Universidad Catholica de Chile. The authors report no other conflicts of interest in this work.

\section{References}

1. Kaniasty K, Norris F. Social support in the aftermath of disasters, catastrophes, and acts terrorism: altruistic, overwhelmed, uncertain, antagonistic, and patriotic communities. Bioterrorism. 2004;3:200229.

2. Camus P, Arenas F, Lagos M, Romero A. Visión histórica de la respuesta a las amenazas naturales en Chile y oportunidades de gestión del riesgo de desastre. Rev Geogr Norte Gd. 2016;64:9-20. doi:10.4067/S0718-34022016000200002

3. Brain R, Mora I. Chile pre y post catástrofe: algunas claves para aproximarse a los desafíos de reconstrucción. En: Emergencia $y$ reconstrucción: El antes y después del terremoto y tsunami del $27 \mathrm{~F}$ en Chile. Aprendizajes en materia habitacional, urbana y de seguros. Santiago: Pontificia Universidad Católica de Chile; 2012:11-38.

4. Barush A. Disaster, vulnerability, and older adults: toward social work response. J Gerontol Soc Work. 2011;54:347-350. doi:10.1080/01634372.2011.582821

5. Cloyd E, Dyer CB. Catastrophic events and older adults. Crit Care Nurs Clin North Am. 2010;22(4):501-513.

6. Aljabri D, Dumitrascu A, Burton MC, et al. Patient portal adoption and use by hospitalized cancer patients: a retrospective study of its impact on adverse events, utilization, and patient satisfaction. $B M C$ Med Inform Decis Mak. 2018;18(1):70.

7. World Health Organization. The World Health Report 2000: Health Systems: Improving Performance. World Health Organization; 2000.

8. Audrefoy J. Desastres y cultura: una aproximación teórica. Revista INVI. 2007;22(60):133-165. Available from: http://revistainvi.uchile. cl/index.php/INVI/article/view/283/928. AccessedAugust 1, 2019.

9. Parmar P, Arii M, Kayden S. Learning from Japan: strengthening US emergency care and disaster response. Health Aff. 2013;32(12):21722178 .

10. Tomio J, Sato H. Emergency and disaster preparedness for chronically ill patients: a review of recommendation. Emerg Med. 2004;6:69-79.

11. Bitterman N. Design of medical devices - a home perspective. Eur J Intern Med. 2011;22(1):39-42. doi:10.1016/j.ejim.2010.09.017

12. Demiris G, Hensel BK. Technologies for an aging society: a systematic review of "smart home" applications. Yearb Med Inform. 2008;17 (1):33-40. doi:10.1055/s-0038-1638580

13. van Den Berg MEL, Maeder AJ. Consumer wearable information and health self management by older adults. Telehealth Our Ageing Soc. 2018;246:42.

14. Puri A, Kim B, Nguyen O, Stolee P, Tung J, Lee J. User acceptance of wrist-worn activity trackers among community-dwelling older adults: mixed method study. JMIR mHealth uHealth. 2017;5(11): e173. doi:10.2196/mhealth.8211

15. Mahoney EL, Mahoney DF. Acceptance of wearable technology by people with Alzheimer's disease: issues and accommodations. Am J Alzheimers Dis Other Demen. 2010;25(6):527-531. doi:10.1177/ 1533317510376944 
16. Lee JA, Choi M, Lee SA, Jiang N. Effective behavioral intervention strategies using mobile health applications for chronic disease management: a systematic review. BMC Med Inform Decis Mak. 2018;18 (1):12. doi:10.1186/s12911-018-0683-x

17. Demiris G, Afrin LB, Speedie S, et al. Patient-centered applications: use of information technology to promote disease management and wellness. A white paper by the AMIA knowledge in motion working group. $J \mathrm{Am}$ Med Inform. 2008;15(1):8-13. doi:10.1197/jamia.M2492

18. Santos JC, Pedrosa T, Costa C, Oliveira JL. Modelling a portable personal health record. Healthinf Third International Conference on Health Informatics, Valencia, Spain. INSTICC-Institute for Systems and Technologies of Information, Control and Communication; 2010:465-468.

19. Kharrazi H, Chisholm R, VanNasdale D, Thompson B. Mobile personal health records: an evaluation of features and functionality. Int $\mathrm{J} \mathrm{Med}$ Inform. 2012;81(9):579-593. doi:10.1016/j.ijmedinf.2012.04.007

20. Kushniruk AW, Borycki EM, Kuwata S, Kannry J. Emerging approaches to usability evaluation of health information systems: towards in-situ analysis of complex healthcare systems and environments. Stud Health Technol Inform. 2011;169:915-919.

21. Schwartz SR, Clouse K, Yende N, et al. Acceptability and feasibility of a mobile phone-based case management intervention to retain mothers and infants from an option $\mathrm{B}+$ program in postpartum HIV care. Matern Child Health J. 2015;19(9):2029-2037. doi:10.1007/ s10995-015-1715-0

22. Westergaard RP, Genz A, Panico K, et al. Acceptability of a mobile health intervention to enhance HIV care coordination for patients with substance use disorders. Addict Sci Clin Pract. 2017;12(1):11. doi:10.1186/s13722-017-0076-y

23. Fearis K, Petrie A. Best practices in early phase medical device development: engineering, prototyping, and the beginnings of a quality management system. Surgery. 2017;161(3):571-575. doi:10.1016/ j.surg.2016.08.052

24. Harte R, Quinlan LR, Glynn L, et al. A multi-stage human factors and comfort assessment of instrumented insoles designed for use in a connected health infrastructure. J Pers Med. 2015;5(4):487-508. doi:10.3390/jpm5040487

25. Davis FD. A technology acceptance model for empirically testing new end-user information systems: theory and results [Doctoral dissertation]. Massachusetts Institute of Technology; 1985

26. Davis FD. Perceived usefulness, perceived ease of use, and user acceptance of information technology. Mis Q. 1989;13:319-340. doi: $10.2307 / 249008$

27. Ma Q, Liu L. The technology acceptance model: a meta-analysis of empirical findings. J Organ End User Com. 2004;16(1):59-72. doi:10.4018/JOEUC

28. Venkatesh V, Thong JY, Xu X. Consumer acceptance and use of information technology: extending the unified theory of acceptance and use of technology. Mis $Q$. 2012;36(1):157-178. doi:10.2307/41410412
29. Kahn JS, Aulakh V, Bosworth A. What it takes: characteristics of the ideal personal health record. Health Aff. 2009;28(2):369-376. doi: $10.1377 /$ hlthaff.28.2.369

30. Stronge AJ, Rogers WA, Fisk AD. Human factors considerations in implementing telemedicine systems to accommodate older adults. $J$ Telemed Telecare. 2007;13(1):1-3. doi:10.1258/1357633077797 01158

31. McGinn C, Grenier S, Duplantie J, et al. Comparison of user groups' perspectives of barriers and facilitators to implementing electronic health records: a systematic review. McGinn et al. BMC Med. 2011;9:46. doi:10.1186/1741-7015-9-46

32. Charness N, Best R, Evans J. Supportive home health care technology for older adults: attitudes and implementation. Gerontechnology. 2016;15(4):233． doi:10.4017/gt.2016.15.4.0 00.00

33. Canales M, Peinado A; Grupos de discusión in Delgado $\mathrm{M}$ and Gutiérrez J. Métodos y técnicas cualitativas de investigación en ciencias sociales. Síntesis; 1995:287-316.

34. Brennan DM, Barker LM. Human factors in the development and implementation of telerehabilitation systems. J Telemed Telecare. 2008;14(2):55-58. doi:10.1258/jtt.2007.007040

35. Demiris G, Rantz MJ, Aud MA, et al. Older adults' attitudes towards and perceptions of 'smart home'technologies: a pilot study. Med Inform Internet Med. 2004;29(2):87-94. doi:10.1080/14639231 0410001684387

36. Harvey JA, Skelton DA, Chastin SF. Acceptability of novel life logging technology to determine context of sedentary behavior in older adults. AIMS Public Health. 2016;3(1):158. doi:10.3934/ publichealth.2016.1.158

37. Mihailidis A, Cockburn A, Longley C, Boger J. The acceptability of home monitoring technology among community-dwelling older adults and baby boomers. Assistive Technol. 2008;20(1):1-12. doi:10.1080/10400435.2008.10131927

38. Thilo FJ, Bilger S, Halfens RJ, Schols JM, Hahn S. Involvement of the end user: exploration of older people's needs and preferences for a wearable fall detection device-a qualitative descriptive study. Patient Prefer Adherence. 2017;11:11. doi:10.2147/PPA. S134792

39. Chung J, Thompson HJ, Joe J, Hall A, Demiris G. Examining Korean and Korean American older adults' perceived acceptability of homebased monitoring technologies in the context of culture. Inform Health Soc Care. 2017;42(1):61-76. doi:10.3109/17538157.2016.11 60244

40. Chaudhuri S. Examining the feasibility and acceptability of a fall detection device [dissertation]. University of Washington Libraries; 2015 .
Patient Preference and Adherence

\section{Publish your work in this journal}

Patient Preference and Adherence is an international, peer-reviewed, open access journal that focusing on the growing importance of patient preference and adherence throughout the therapeutic continuum. Patient satisfaction, acceptability, quality of life, compliance, persistence and their role in developing new therapeutic modalities and compounds to optimize clinical outcomes for existing disease states are major areas of interest for the journal. This journal has been accepted for indexing on PubMed Central. The manuscript management system is completely online and includes a very quick and fair peer-review system, which is all easy to use. Visit http:// www.dovepress.com/testimonials.php to read real quotes from published authors. 